\title{
Divisionalization in Contests
}

\author{
Stexen Huck, Kai A. Konrad and Wieland Müller"
}

J anuary 31,2000

\begin{abstract}
A bstract
To be represented by more than one contestant in a contest has advantages and disadvantages. This paper determines the conditions under which it is favorable to send several agents into the contest.
\end{abstract}

J EL classi..cation numbers: D44, L11, L13

K eywords: Divisionalization, contests, delegation

\section{Introduction}

In many contest environments, the total number of players is limited, but players can choose between sending one or several agents to compete in a contest. For instance, in car races teams can send one or two cars into the race. In $R \& D$ contests, ..rms may have just one research laboratory or may divisionalize their research activities, installing several independent research units. More importantly, in markets in which price competition is exectively ruled out and all competition occurs via upfront sunk sales exorts, ..rms may decide to have only one sales department, or they may divisionalize and choose competing sales agencies. T wo examples are the market for prescription drugs in countries in which price competition for drugs is exectively eliminated due to the indirect payment arrangements of the health care system, and the highly regulated pre-1994 insurance market in many European countries. ${ }^{1}$ There seems to be

\footnotetext{
${ }^{x}$ Royal Holloway College, Free University of B erlin and Humboldt University, respectively. Correspondence to $\mathrm{K}$ ai $\mathrm{A}$. Konrad, Department of E conomics, Free University of B erlin, B oltzmannstrasse 20, D-14195 Berlin, e-mail: kai.konrad@wiwiss.fu-berlin.de.

${ }^{1}$ For a characterization of the European insurance market see R ees and K essner (1999).
} 
little evidence for divisionalization in the market for prescription drugs, whereas, in the insurance market, some big ..rms owned several smaller insurance companies who contested for customers with each other and with the rest of the market and some companies even employed a number of sales agencies that cannibalized on each other.

In this paper we address the question whether divisionalization is pro..table. We disregard aspects such as intra-..rm governance problems and competing divisions as an internal incentive system or economies of scale and other cost exects and concentrate on the strategic aspects that relate to the actual contest. There are three contest aspects. First, divisionalization increases the number of competitors. Typically this will reduce the rents earned by all contestants. Second, if a ..rm sends several competing agents into the contest, this ..rm's share in the aggregate rent is increased. Third, if a ..rm sends several agents into the contest and pays for their exorts, the divisions partially cannibalize on each other. The ..rm may take this into account and may reduce competition between the divisions.

In a related context, Baye, Crocker and J u (1996) considered whether ..rms can gain from divisionalization in a Cournot oligopoly. The divisionalizing ..rm gains and overall pro..ts in this industry are reduced. Divisionalization in contests has similar exects as regards total pro..ts, but whether the divisionalizing ...rm gains depends on the number of competitors and on the characteristics of the contest.

\section{Contests}

Let $\mathrm{N}$ be the set of ..rms, with \# $\mathrm{N}=\mathrm{n}$ : Suppose that each of these ..rms has one contestant in a contest that makes an exort to win some prize of size B. E ach contestant i chooses contest exort $x_{i} 2[0 ; 1$ ) which cannot be recovered, whether the contestant wins or not. Contest exorts determine contestants' probabilities q of winning the prize, according to a contest success function

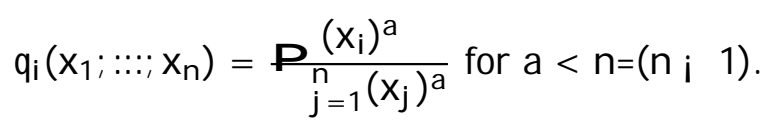

This function has been suggested by Tullock (1980) and axiomatized by Skaperdas (1996). The coec cient a in (1) measures how much the contest outcome can be inłuenced by contest exort, and is called the discriminatory power of the contest success 
function. For instance, if a ! 0 , each contestant has the same chance of winning, irrespective of contest exorts. If, instead, a ! 1 , (1) approaches a contest success function in which the contestant who makes the highest exort wins for sure. We limit the discriminatory power to a $2\left[0 ; \frac{n}{n_{i} 1}\right)$ in order to have well-behaved optimization problems with equilibria in pure strategies and ..rst-order conditions characterizing these equilibria. $^{2}$

Firms are risk neutral. Suppose there is no incentive problem between ..rms and contestants. They maximize ..rms' payous

$$
1 / 4=q B i x_{i}
$$

Firm i wins the prize B with probability $q$ and its contestant spends contest exort equal to $x_{i}$. The ..rst-order condition for a payo maximum and symmetry can be used to calculate the contest equilibrium exorts

$$
x^{\mathbb{a}}(\mathrm{n})=\frac{\mathrm{aB}(\mathrm{n} \mathrm{i} 1)}{\mathrm{n}^{2}}
$$

with $\mathrm{n}$ being the number of contestants. The equilibrium probability of winning is $1=n$ for each contestant, yielding the equilibrium payoxs as

$$
1 / \frac{\alpha}{4}(n)=\frac{B}{n} i \frac{a B\left(n_{i} 1\right)}{n^{2}} .
$$

\section{Fully non-cooperative divisions}

Suppose ..rm $\mathrm{n}$ decides to divisionalize, i.e., to send two divisions to the contest and provides each division with incentives to maximize payou as in (2). Divisionalization increases the set of contestants from $\mathrm{f} 1 ; \ldots ; ; \mathrm{ng}=\mathrm{N}$ to $\mathrm{f} 1 ; \ldots ; \mathrm{n}_{1} ; \mathrm{n}_{2} \mathrm{~g}=\mathrm{N} \mathrm{d}$. We denote $f 1 ;: . ;(n ; 1) g$ ' $S$ and $f n_{1} ; n_{2} g$ ' $D$. The latter is the set of divisions of ..rm n. Divisionalization is pro..table if the equilibrium payox of the two divisions in the contest with $n+1$ participants exceeds the payox of one participant in a contest with $n$ participants. This is the case if $1 / \frac{\alpha}{4}(n)<2 \frac{1 / \alpha}{4}(n+1)$, or, using (4), if

$$
\frac{(n+1)(n ; 1) n}{n^{3} i n^{2}+n+1}>a
$$

\footnotetext{
${ }^{2}$ For the equilibrium (in mixed strategies) for the case with $a>n=n ; 1$ ) see Baye, Kovenock and deVries (1994).
} 
Denote the left hand side of $(5)$ by $a_{0}(n)$ and note that $a_{0}(n) 2\left(0 ; \frac{n}{n_{i} 1}\right)$. Hence,

Proposition 1 For a given total number $n$ of ..rms there is a critical discriminatory power $a_{0}(n) 2\left(0 ; \frac{n}{n_{i} 1}\right)$ such that divisionalization by one ..rm is pro..table for this ..rm if and only if $a<a_{0}(n)$.

Intuitively, if the discriminatory power is very small, e.g., close to zero, then total exort becomes negligible in comparison to the prize. Accordingly, each ..rm earns almost $B=n$ if it does not divisionalize, and almost $2 B=(n+1)$ if it divisionalizes.

We also note that divisionalization reduces total industry pro..ts, as $n^{1 / 8}(n)$ in (4) is declining in $\mathrm{n}$.

\section{Cooperative divisions}

If divisionalization occurs within a ..rm, the ..rm's divisions' exorts may be coordinated by a higher level decision making unit that takes into account that the contest exort in one division also reduces the other division's probability of winning.

In this case the total exect of divisionalization can be obtained analytically in two steps. First, suppose the new divisions behave non-cooperatively. This exect has been determined in section 3. We call this the team size exect because it is based on the increased number of ..rm n's contestants. Second, divisions' choices of exort may be coordinated. We call this the collusion exect. To analyse the collusion exect we start with a situation in which all contestants in $\mathrm{N}^{\mathrm{d}}$ behave non-cooperatively and ask if the two divisions $n_{1}$ and $n_{2}$ can increase their joint pro..ts if they choose their exorts cooperatively.

If divisions maximize ..rm pro..t $\left(q_{1}+q_{2}\right) B$ i $x_{n_{1}} i x_{n_{2}}$, they take into account $\frac{a_{a}}{a_{j}}<0$ for $i \epsilon j$, and therefore, coordination makes them reduce their exort compared to $x^{\bowtie}(n+1)$. This decrease is anticipated by contestants $k 2$ S. Let $\gg(x)$ be the symmetric non-cooperative equilibrium exort choices of divisions $n_{i} 2 D$, for given exort choices $x_{j}=x$ for all $j 2 S$, and similarly, $\stackrel{1}{\gg}(x)$ the symmetric non-cooperative equilibrium exort choices of all contestants j $2 \mathrm{~S}$ for given exort choices $\mathrm{x}_{n_{i}}=\mathrm{x}$ for $\mathrm{n}_{\mathrm{i}} 2 \mathrm{D}$. These functions could, in principle, be obtained analytically from solving the 
..rst-order conditions for fully non-cooperative ..rms,

$$
\frac{a x_{i_{3}}^{a i}{ }_{j 2 N d n f, i g} x_{j}^{a}}{P} B \text { i } 1=0,
$$

for $x$ as a function of $x$, with $x$ the non-cooperative exort level chosen by all contestants in $D$ for given uniform exort levels $x$ by all contestants $j 2 S$, and vice versa for $x$ as a function of $x$. The intersection of these two curves characterizes the Nash equilibrium with $\left.x_{i}=x_{j}=x^{a}(n+1)=a B n=n+1\right)^{2}$ for all $i ; j 2 N^{d}$ by (3). For i $2 S$, (6) can be rewritten as ax ${ }^{a}{ }^{1}\left[(n ; 2) x^{a}+2 x^{a}\right] B=\left[(n ; 1) x^{a}+2 x^{a}\right]^{2}$. At the equilibrium value $x^{\alpha}$, by dixerentiating this condition totally and making use of $(3)$, the slope of $\stackrel{1}{\gg}(x)$ can be obtained as

$$
\gg(x)=\frac{2 a(n i 1)}{i n^{2} i n i 2 a+2 n a} .
$$

Lemma $1 \stackrel{1}{\gg}(x)<0$ and $\lim _{a} 0^{\frac{1}{》}} 9(x)=0$ at $x=x=x^{x}(n+1)$.

For a proof of Lemma 1 observe that the numerator of (7) is positive and the denominator of (7) is negative for $a \cdot \frac{n}{n_{i} 1}$ and converges toward $i 1$ for $a ! 0$. The intuition for the limit property in Lemma 1 is as follows. By (6), for a ! 0 each contestant's marginal bene.t. from exort becomes in..nitely small. Hence, a contestant would not like to spend much, even if other contestants spend huge amounts.

Using the envelope theorem and the fact that $\frac{@ / 4}{\varrho_{k}}=i \frac{1}{n}$ for $i \quad \xi k$ at the fully non-cooperative Nash equilibrium, the pro..t is higher for each division if they jointly reduce their contest exort $x$ starting from $x=x=x^{\alpha}(n+1)$ if

$$
\mathrm{i} \frac{\mathrm{d}^{1} / \mathfrak{t}}{\mathrm{dx}}=\frac{1}{\mathrm{n}}\left(1+(\mathrm{n} ; \quad 1)^{\frac{1}{\gg}}(\mathrm{x})\right)>0
$$

A joint decrease in their exorts increases their pro.ts if the mutual direct exect of reduced exort among the divisions outweighs the equilibrium reactions by all other contestants. The inequality in (8) to be ful..lled is a necessary condition for collusion to be pro..table. It resembles the condition that has been derived in Gaudet and Salant (1991) who consider merger in Cournot competition. The pro..tability exect of collusion is ambiguous in general. However, by Lemma 1, we have 
Proposition 2 A joint marginal anticipated reduction in contest exort among divisions is pro..table if (8) holds. (8) holds if the discriminatory power of the contest is suф̧ ciently low.

The intuition for the result in Proposition 2 is as follows. Collusion on contest exort leads to a reduction in exort for the set of colluders. If this reduction in exort does not trigger an increase in other contestants' exorts, collusion is bene..cial. As has been shown in Lemma 1, other contestants react by increases in their exort, but this reaction is very moderate if the discriminatory power of the contest is suф ciently low.

\section{Conclusions}

In this paper we consider the pro..tability of divisionalization in contests. We distinguish between a team size exect and a collusion exect and show that with high discriminatory power the team size exect of divisionalization reduces pro..ts and the collusion exect is ambiguous, but both exects increase pro..ts if the discriminatory power in the contest is low.

\section{References}

B aye, M.R., K.J . Crocker and J . J u, 1996, Divisionalization, franchising, and divestiture incentives in oligopoly, A merican Economic R eview, 86, 223-36.

B aye, M.R., D. K ovenock, and C.G. deVries, 1994, The solution to the Tullock rentseeking game when $\mathrm{R}$ is greater than 2 : mixed-strategy equilibria and mean dissipation rates, Public Choice, 81, 363-380.

Gaudet, G., and S.W. Salant, 1991, Increasing the pro..ts of a subset of ..rms in oligopoly models with strategic substitutes, A merican E conomic R eview, 81, 658-665.

Rees, R., and E. Kessner, 1999, E uropean insurance markets, regulation and eф ciency, E conomic Policy, 29, 363-397.

Skaperdas, S., 1996, Contest success functions, E conomic Theory, 7, 283-290.

Tullock, G., 1980, E $₫$ cient rent seeking, in: J .M. Buchanan, R.D. Tollison and G. Tullock, eds., Toward a theory of rent-seeking society (Texas A\&M University Press, College Station) 97-112. 